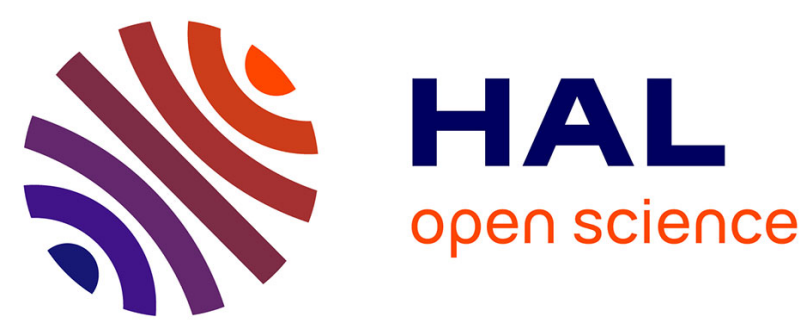

\title{
Méthode d'évaluation de la biocontamination de surface des matériaux cimentaires par microscopie confocale à balayage laser
}

Caroline Chagnot, Chao Shen, Charlotte Munzer, Julien J. Deschamps, Françoise Feugeas, Thierry T. Meylheuc

\section{To cite this version:}

Caroline Chagnot, Chao Shen, Charlotte Munzer, Julien J. Deschamps, Françoise Feugeas, et al.. Méthode d'évaluation de la biocontamination de surface des matériaux cimentaires par microscopie confocale à balayage laser. Matériaux \& Techniques, 2015, 103 (2), 10.1051/mattech/2015027 . hal02635775

\section{HAL Id: hal-02635775 \\ https://hal.inrae.fr/hal-02635775}

Submitted on 27 May 2020

HAL is a multi-disciplinary open access archive for the deposit and dissemination of scientific research documents, whether they are published or not. The documents may come from teaching and research institutions in France or abroad, or from public or private research centers.
L'archive ouverte pluridisciplinaire HAL, est destinée au dépôt et à la diffusion de documents scientifiques de niveau recherche, publiés ou non, émanant des établissements d'enseignement et de recherche français ou étrangers, des laboratoires publics ou privés. 


\title{
Méthode d'évaluation de la biocontamination de surface des matériaux cimentaires par microscopie confocale à balayage laser
}

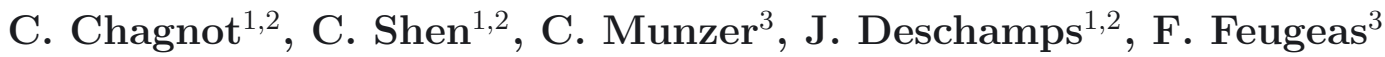 \\ et T. Meylheuc $c^{1,2,4}$
}

Reçu le 25 février 2015, accepté le 6 mai 2015

\begin{abstract}
Résumé - La biodéterioration des matériaux cimentaires tels que les bétons est le plus souvent liée à la formation de structures microbiennes complexes appelé biofilm. Ce développement microbien, en dégradant le matériau, génère des coûts financiers et énergétiques importants, ainsi qu'un risque potentiel pour la santé humaine. De nombreux travaux ont étudiés la formation des biofilms sur des surfaces abiotiques simples (polymères, acier, ciment...) cependant, jusqu'à présent, l'étude à l'échelle microscopique de la structure des biofilms se développant à la surface des bétons se heurtait à différents obstacles liés à la taille des échantillons et à l'hétérogénéité du matériau. Notre étude a permis de développer une nouvelle méthode d'analyse par microscopie confocale à balayage laser, la méthode SMART (Système de Mesure Appliqué au Recouvrement des biofilms cimenTaires), permettant de mesurer l'hétérogénéité des biofilms à la surface des matériaux cimentaires et de quantifier cette biocontamination par le calcul du biovolume pondéré de ces structures tridimensionnelles.
\end{abstract}

Mots clés : Biocontamination / bactérie / biofilm / bio réceptivité / surfaces cimentaires

\begin{abstract}
Evaluation method of biocontamination surface of cementitious materials by confocal Laser scanning microscopy. Biodeterioration of cementitious materials such as concrete is related to the formation of complex microbial structures called biofilm. This microbial growth, degrading the material causes of significant financial and energetics costs and a potential risk to human health. Many studies have investigated the formation of biofilms on simple abiotic surfaces (polymers, steel, cement...), however, the microscopic study of biofilm structure on the surface of concrete was blocked by various obstacles related to the sample size and heterogeneity of the material. Our study developed a new method of confocal laser scanning microscopy, the SMART method (Système de Mesure Appliqué au Recouvrement des biofilms cimenTaires) permitting to measure the heterogeneity of biofilms on the surface of cementitious materials and quantify biocontamination by precise calculation of the weighted biovolume.
\end{abstract}

Key words: Biocontamination / bacteria / biofilm / bio receptivity / cementitious materials

\section{Introduction}

Le béton est le matériau manufacturé le plus utilisé au monde principalement dans les domaines du bâtiment et du génie civil, il représente, en terme de production, 6 milliards de $\mathrm{m}^{3}$ par an (http://www.planetoscope. com). Le béton est un matériau cimentaire principalement composé d'un liant à base de ciment, d'eau et de granulats (gravillons, sables...). D'autres éléments peuvent être utilisés tels que des adjuvants permettant d'apporter de nouvelles propriétés au matériau ou aux armatures en acier ou en carbone dans le cas des bétons armés. Comme tous les matériaux, les matériaux cimentaires sont sujets à la dégradation liée au temps et à l'environnement. La

\footnotetext{
1 INRA, UMR 1319 Micalis, 25 avenue de la République, 91300 Massy, France

thierry.meylheuc@jouy.inra.fr

2 AgroParisTech, UMR Micalis, 25 avenue de la République, 91300 Massy, France

3 ICube UMR 7357, INSA de Strasbourg, CNRS, 24 boulevard de la Victoire, 67084 Strasbourg Cedex, France

4 INRA, Centre de recherche Massy, UMR 1319 MicAliS, 91300 Massy, France
} 
maintenance et le remplacement des matériaux endommagés entraînent des coûts financiers et énergétiques importants [1]. De nombreuses raisons biologiques et chimiques peuvent expliquer ce phénomène, parmi elles se trouvent les dégradations liées à l'installation des biofilms bactériens.

Les biofilms sont des structures composées de microorganismes adhérant entre eux et d'une matrice adhésive et protectrice. Généralement, en milieux aqueux, ils peuvent se développer sur n'importe quel type de surface naturelle ou artificielle. La formation de biofilms est un comportement classique chez les bactéries, ils peuvent être composés d'une ou plusieurs espèces bactériennes en interaction. Dans un contexte industriel, des études ont montré que toute niche ou anfractuosité pouvait favoriser l'adhésion voir le piégeage de germes d'altération ou pathogènes (Listeria, Legionella, coliformes fécaux, etc.) [2-4]. De plus, les micro-organismes présents dans ces consortia microbiens sont englués dans une matrice plus ou moins cohésive qui compose également le biofilm, matrice qui forme une couche protectrice pour les bactéries et inhibe fortement l'action des biocides. Cette protection dont bénéficient les cellules en biofilm constitue donc un risque élevé pour la santé humaine et animale lorsqu'il s'agit de germes pathogènes. Cependant cette capacité à coloniser les surfaces peut également être utilisée à des fins bénéfiques et protectrices (biofilms positifs) en prévention contre la contamination microbienne (Ex : inoculation de biofilms positifs dans les locaux d'élevage de volailles dans l'industrie agroalimentaire). La biodégradation des matériaux cimentaires ainsi que la composition bactérienne des biofilms ont fait l'objet de plusieurs études [5-9]. Plusieurs causes de détérioration peuvent être imputés aux bactéries tels que la production d'acides organiques (Ex : lactique, acétique) et du dioxyde de carbone extrêmement corrosive pour les matériaux cimentaires ou encore de la transformation du sulfure d'hydrogène en acide sulfurique. La capacité des bactéries à pénétrer dans les microfissures des ciments pourrait également être source de dégradation du matériau [6]. Cependant, de nombreux verrous technologiques limitent l'étude microscopique structurale des biofilms se développant sur des surfaces cimentaires. Ils sont principalement liés à l'hétérogénéité de la structure de ce type de matériau. Les paramètres structuraux des biofilms sont indispensables afin de comprendre et de mieux maîtriser la formation de ceuxci sur les surfaces cimentaires dans le but de corréler les caractéristiques des biofilms en fonction des types bactériens colonisateurs et des caractéristiques des supports. Pour cela, le développement d'une méthode d'analyse permettant l'étude microscopique des biofilms sur ce type de matériaux est indispensable. En effet, les méthodes classiques de quantification des biofilms sont le plus souvent appliquées de manière globale (par simple moyenne de tous les champs observés). Ces images sont directement analysées par des logiciels de reconstruction et de quantification (Ex : Phlip, Imaris) sans qu'au préalable ne soit appliquée une pondération (basée sur échantillonnage prédéfini) sur la représentativité des images obtenues. Afin d'optimiser ces méthodes, nous proposons une nouvelle méthodologie pour l'analyse de biofilms hétérogènes par microscopie confocale à balayage laser permettant de caractériser et de quantifier plus finement la biocontamination d'échantillons cimentaires à l'échelle microscopique.

\section{Matériels et méthodes}

\subsection{Souches et cultures bactériennes}

Pour cette étude, six souches bactériennes ont été sélectionnées pour leur capacité à former des biofilms dans différents environnements naturels (Tab. 1) [10-12]. Ces bactéries ont été conservées en cryotubes à $-80^{\circ} \mathrm{C}$ dans $20 \%$ de glycérol (v/v). Les cellules congelées ont été mises en préculture puis en culture dans un milieu nutritif complexe, le milieu TSB (Tryptic Soy Broth, Biomérieux, France), à $25{ }^{\circ} \mathrm{C}$ sans agitation durant $24 \mathrm{~h}$, afin d'atteindre la phase stationnaire de croissance des bactéries, phase à laquelle le taux de croissance est nul (nombre de nouvelles cellules $=$ nombre de cellules qui meurent) et où la concentration bactérienne résultante est stable. Au jeune âge, le matériau cimentaire présente un $\mathrm{pH}=13$, en fonction de ses conditions de cure et de vieillissement, le $\mathrm{pH}$ peut diminuer jusqu'à 9 (carbonatation) [13]. Cette diminution de $\mathrm{pH}$ peut influencer la capacité des bactéries à adhérer et à former des biofilms à leur surface. Le milieu de culture a été ajusté à différents $\mathrm{pH}$ compris entre 7 et 13 , avec une solution de chlorure de potassium (KCL) à $3 \mathrm{M}$, afin de réaliser les tests de sensibilité des souches sélectionnées aux pH basiques.

\subsection{Surfaces d'étude}

\subsubsection{Surface de polystyrène}

La formation des biofilms a été effectuée dans des plaques de microtitrage en polystyrène 12 puits $\left(\mu\right.$ clear ${ }^{\circledR}$ base, Greiner Bio-one, France), adaptées à l'imagerie de fluorescence de haute résolution [14].

\subsubsection{Pastilles cimentaires}

La réalisation des pastilles de ciment a été faite selon la méthode de Munzer et al. [15] en accord avec la norme NF EN 196-3. Les pâtes de ciment composées d'eau et de ciment avec un rapport $\mathrm{E} / \mathrm{C}$ de 0,315 , ont été coulées dans des microplaques 12 puits afin de réaliser des pastilles de taille et de forme constantes et maîtrisées, compatibles avec les observations en microscopie confocale (diamètre $22 \mathrm{~mm}$ et d'épaisseur $3 \mathrm{~mm}$ ). Le pH de surface des pastilles de ciment immergées dans l'eau ou dans le milieu TSB a été mesuré à l'aide d'une électrode de surface (SCHOTT instrument L39, SI analytics). 
Tableau 1. Souches bactériennes utilisées dans cette étude.

Table 1. Bacterials strains used in this study.

\begin{tabular}{ccc}
\hline Espèce & Souche & Source \\
\hline Bacillus thuringiensis $(B t)$ & 407 & Houry et al. [10] \\
Escherichia coli $(E c)$ & K12 779P-Ec & Souche de référence \\
Enterrococcus faecalis $(E f)$ & ATCC 29302 & www. atcc.org \\
Pseudomonas fluorescens $(\mathrm{Pf})$ & SBW25 ViscA 0931 & Koza et al. [11] \\
Staphylococcus aureus $(\mathrm{Sa})$ & ATCC 6538 & www.atcc.org \\
Stenotrophomonas maltophilia $(\mathrm{Sm})$ & 114N-Sm & Lacroix-Gueu et al. [12] \\
\hline
\end{tabular}

\subsection{Formation des biofilms bactériens}

\subsubsection{Surface polystyrène}

À partir d'une préculture de $24 \mathrm{~h}$, une culture de $24 \mathrm{~h}$ a été réalisée après dilution au $1 / 100^{\mathrm{ème}}$. La suspension bactérienne a été ajustée à une $\mathrm{DO}=0,2$, puis dans des plaques de microtitrage en polystyrène 12 puits $\left(\mu\right.$ clear ${ }^{\circledR}$ base, Greiner Bio-one, France), $1 \mathrm{~mL}$ a été réparti dans chaque puits et incubé $2 \mathrm{~h}$, à $25^{\circ} \mathrm{C}$. Après incubation, la suspension bactérienne a été éliminée par pipetage et les puits lavés à trois reprises avec $1 \mathrm{~mL}$ d'eau physiologique pour éliminer les cellules non adhérentes. Par la suite, les bactéries adhérentes ont été incubées dans $1 \mathrm{~mL}$ de milieu TSB à $25^{\circ} \mathrm{C}$. Après 3 jours, le milieu était éliminé par pipetage. $200 \mu \mathrm{L}$ d'un mélange $(\mathrm{v} / \mathrm{v})$ de fluorophores marqueurs des acides nucléiques composé de SYTO ${ }^{\circledR} 9$ et d'IP (iodure de propidium) dilué au $1 / 100^{e ̀ m e}$ ont été ajoutés dans chaque puits, durant 2 min à l'obscurité [16]. Le SYTO ${ }^{\circledR} 9$ dont les qualités hydrophiles le rende perméable aux membranes et parois cellulaires lui permettant de marquer l'ensemble des cellules viables (intégrité membranaire conservée) ou endommagées (membrane perméabilisée), en se fixant aux acides nucléiques (fluorescence vert). L'IP, imperméable aux membranes cellulaires, permet quant à lui de révéler uniquement les acides nucléiques des cellules endommagées (fluorescence rouge). Ainsi il est possible de différencier les cellules viables des cellules lysé composant le biofilm bactérien. Pour chaque souche bactérienne, les biofilms ont été réalisés en triplicats biologiques et techniques, à savoir trois solutions bactériennes distinctes sont utilisées pour former les biofilms de l'étude (triplicat biologique). Chacune des trois suspensions est repartie dans trois puits différents (triplicat technique). La représentation graphique des résultats correspond à la moyenne des triplicats biologiques (pondéré par les triplicats techniques) et leurs variations calculées (écart type) sont représentées sous la forme de barres d'erreur.

\subsubsection{Surface cimentaire}

À la surface des pastilles cimentaires, les biofilms ont été réalisés dans les conditions précédemment décrites de préculture et de culture bactérienne. Chaque pastille de ciment a été déposée dans une boite de Pétri $(35 \times 15 \mathrm{~mm})$ et immergée dans $4 \mathrm{~mL}$ de suspension bactérienne, ajustée à une $\mathrm{DO}=0,2$ pendant $2 \mathrm{~h}$ à
$25{ }^{\circ} \mathrm{C}$. Les bactéries non adhérentes étaient éliminées par deux bains de rinçages successifs d'eau physiologique. La croissance des biofilms a ensuite été réalisée dans $4 \mathrm{~mL}$ de milieu TSB stérile pendant 3 jours à $25^{\circ} \mathrm{C}$. Les pastilles cimentaires ont été prélevées et colorées avec $200 \mu \mathrm{L}$ du mélange SYTO9/IP précédemment décrit. Cette expérimentation a été réalisée en triplicat pour chacune des souches bactériennes sélectionnées.

\subsection{Acquisition et analyse d'image en microscopie confocale à balayage laser (MCBL)}

\subsubsection{Observations}

Les observations microscopiques ont été réalisées à l'aide d'un microscope confocal à balayage laser inversé, Leica SP2 AOBS (MCBL, Leica Microsystems, France) au sein de la plate-forme de microscopie INRA MIMA2 (http://voxel.jouy.inra.fr/mima2). Les pastilles de ciment ont été déposées sur une lamelle de verre, la face «biofilm » orientée vers l'objectif, sur deux espaceurs $(0,3 \mathrm{~mm})$, évitant ainsi l'écrasement des biofilms. Les pastilles ainsi que les microplaques de polystyrène ont ensuite été montées sur la platine motorisée du microscope et observées à l'aide d'un objectif $\times 10 / 0.3 \mathrm{~s}$ (Leica 506505). Les cellules bactériennes ont été excitées à l'aide d'un laser argon révélant les marquages fluorescents du SYTO9 (Excitation/Emission 488/498 nm) et de l'IP (Excitation/Emission 488/595 nm). L'hétérogénéité des biofilms a été évaluée par prise d'images sur 25 zones préalablement définies (coordonnées) réparties à la surface de chaque échantillon (puits ou pastille) (Fig. 1). Le champ d'observation correspondant à chaque image est de $1,16 \times 1,16 \mathrm{~mm}$. Le biovolume a été obtenu en réalisant des séries d'images en Z (ou stack d'images) selon un pas de $10 \mu \mathrm{m}$ ceci sur 5 zones représentatives du biofilm, sélectionnées à partir des images précédemment obtenues.

\subsubsection{Analyse d'image}

Une analyse d'image permettant après binarisation de calculer le pourcentage de recouvrement des bactéries sur la surface étudiée a été réalisée sur les 25 champs observés (logiciel IMARIS v7.0 software, Bitplane, Switzerland). Le biovolume, volume global d'un biofilm (en $\mu \mathrm{m}^{3}$ ) permettant d'estimer sa biomasse totale, est estimé à partir 


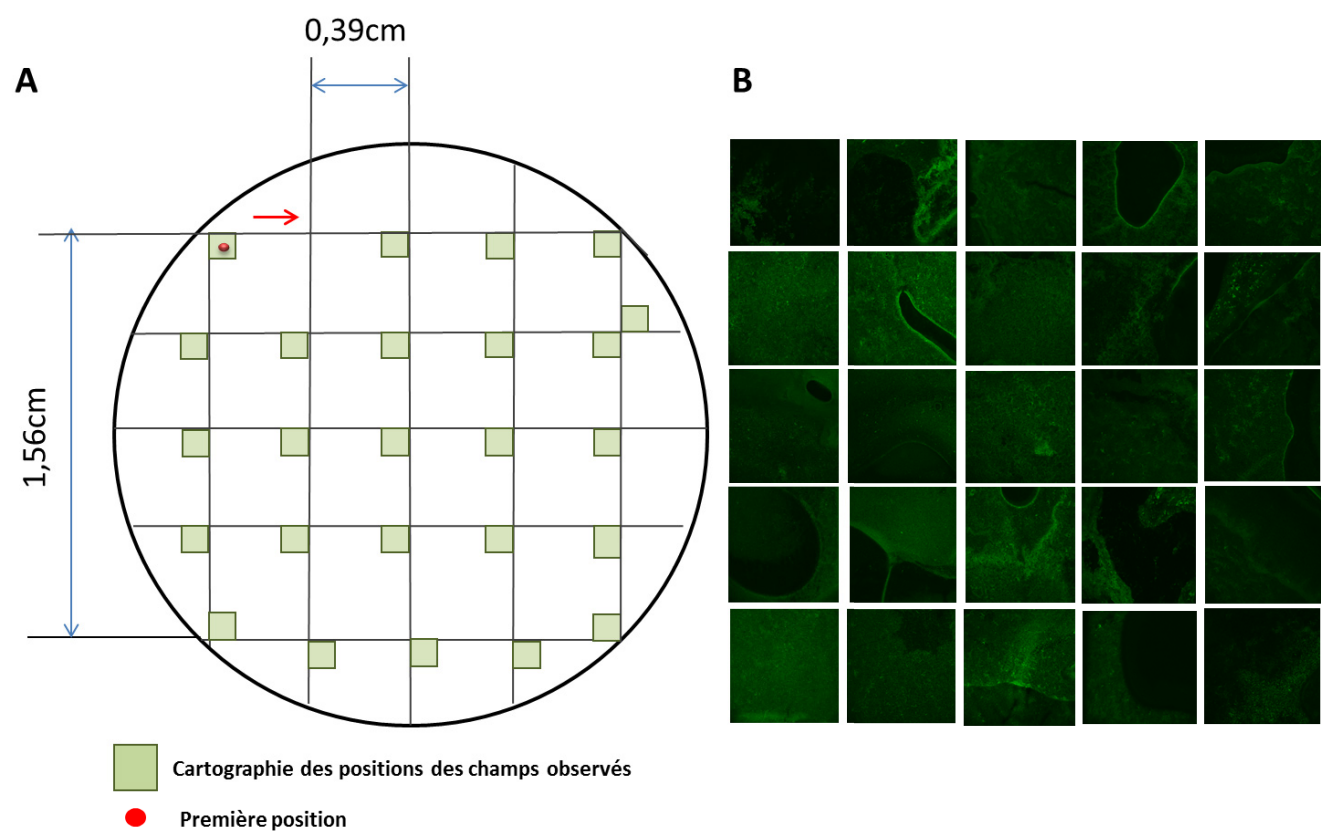

Fig. 1. Cartographie et observation d'un biofilm « cimentaire $»$.

(A) Schémas des coordonnées correspondant aux 25 champs d'acquisitions sélectionnés pour l'étude de l'hétérogénéité des biofilms bactériens à la surface d'une pastille de ciment. (B) Exemple d'une série d'images réalisées par microscopie confocale à balayage laser (MCBL) sur un biofilm d'Escherichia coli $\mathrm{K} 12\left(72 \mathrm{~h}\right.$ à $\left.25^{\circ} \mathrm{C}\right)$.

Fig. 1. Cartography and observation of a "concrete biofilm".

(A) Schema of the coordinates corresponding to the 25 acquisitions selected for the study of biofilms heterogeneity on the concrete pellet surface. (B) Example of pictures series of Escherichia coli K12 biofilm (72 $h$ at $25{ }^{\circ} \mathrm{C}$ ) by confocal Laser Scanning Microscopy (CLSM).

d'un stack d'images en Z et analysés à l'aide du logiciel PHLIP fonctionnant sous Matlab (http://phlip. sourceforge.net/phlip-ml) [17]. Le biovolume a été défini en tenant compte du nombre de pixels de chaque image en Z multiplié par le volume de voxel, qui est le produit de la taille des pixels au carré et de la taille de pas de balayage [18]. Les analyses statistiques (ANOVA) a été effectuées en utilisant le logiciel Statgraphics v6.0 (Manugistics, Rockville, Etats-Unis). Une valeur de $P$ associée au test de Fisher est calculée après comparaison des données, pour une valeur $P<0,05$ les données sont considérées comme significativement différentes.

\section{Resultats et discussions}

\subsection{Biofilms sur surface polystyrène}

Afin d'étudier la formation des biofilms à la surface des ciments, une première étape a consisté en une sélection de souches bactériennes issues d'environnements naturels, capables de former des biofilms. Sur les surfaces en polystyrène, les six souches bactériennes choisies ont toutes formé des biofilms reproductibles. L'épaisseur des biofilms obtenus variait de 35 à $60 \mu \mathrm{m}$ (Fig. 2). Au cours de la croissance du biofilm, la mortalité cellulaire augmente inévitablement suite entre autre à l'appauvrissement du milieu au cours du temps, cependant une plus forte mortalité cellulaire a été observée pour les souches Stenotrophomonas maltophilia 114N-Sm (Sm 114N-Sm) et Enterrococcus faecalis ATCC 29302 (Ef ATCC 29302). Ces souches n'ont donc pas été retenues pour la suite de l'étude. De plus, l'analyse de la projection d'ombre projetée permet de mettre en évidences la répartition du biofilm ainsi que sa densité. Ainsi, les biofilms formés par les souches Pseudomonas fluorescens SBW25 ViscA (Pf SBW25 ViscA) et Staphylococcus aureus ATCC 6538 (Sa ATCC 6538), présentaient une forme aérée (biofilm de faible densité) avec des biovolumes similaires de $1,5 \times 10^{8} \mu \mathrm{m}^{3}$. Les souches Escherichia. coli K12 (Ec K12) et Bacillus thuringiensis 407 (Bt 407) ont formé des biofilms moins épais mais plus denses, avec un biovolume variant de $1,5 \times 10^{8} \mu \mathrm{m}^{3}$ à $0,8 \times 10^{8} \mu \mathrm{m}^{3}$. Sur la base des caractéristiques tridimensionnelles (densité, épaisseur) observées, les trois souches, Pf SBW25 ViscA, $E c$ K12 et Bt 407 ont été retenues pour leur capacité à former des biofilms à la fois reproductibles et présentant une faible mortalité.

\subsection{Biofilms sur surface cimentaire}

\subsubsection{Paramètres influant sur la formation des biofilms}

La formation des biofilms bactériens est dépendante d'un grand nombre de paramètres dont certains, comme 


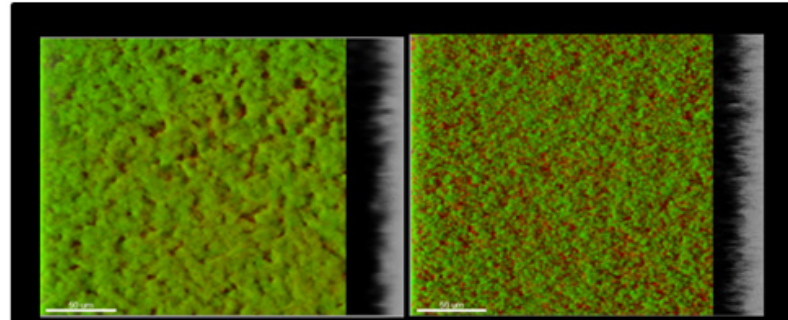

Sm. $114 \mathrm{~N}-\mathrm{Sm}$

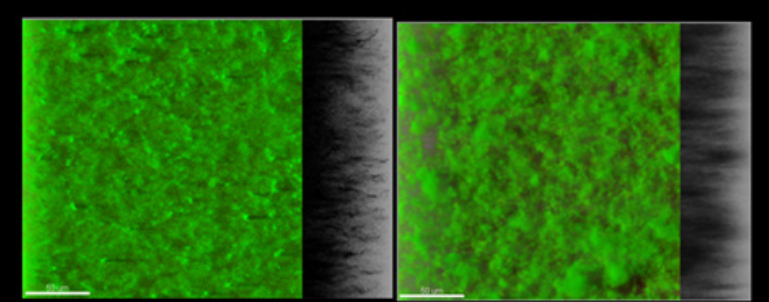

Pf SBW25 viscA

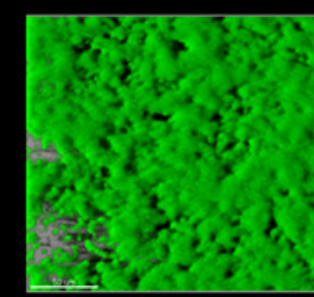

Bt 407
Sa ATCC 6538

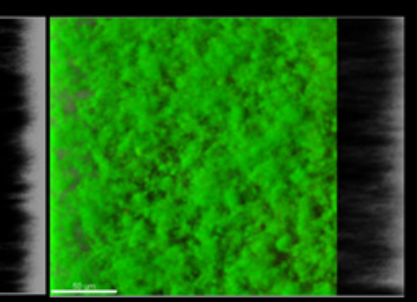

Ec K12
Fig. 2. Formation de biofilms bactériens sur des surfaces en polystyrène.

Diversité structurale des biofilms des souches $S m$ 114N-Sm, Ef ATCC 29302, Pf SBW25 ViscA, Sa ATCC 6538Ec K12 Bt 407 observé à la surface de microplaques de polystyrène. Les images correspondent à la projection tridimensionnelle du biofilm formé par les six souches obtenues en microscopie confocale à partir d'une série images en $\mathrm{Z}$ analysées par le logiciel IMARIS. Les images présentent une vue aérienne de la structure du biofilm avec la projection d'ombre virtuelle sur la droite. La barre d'échelle correspond à $50 \mu \mathrm{m}$.

Fig. 2. Formation of bacterial biofilm on polystyrene surfaces. Structural biofilms diversity of Sm 114N-Sm, Ef ATCC 29302, Pf SBW25 ViscA, Sa ATCC 6538Ec K12 Bt 407, observed on polystyrene microplates surface. Images correspond to threedimensional projection of the six strains biofilms obtained from $Z$ confocal images series using IMARIS software. Images present an serial view of biofilm structure with the virtual shadow projection on the right. Scale bar correspond to $50 \mu \mathrm{m}$.

la température ou le $\mathrm{pH}$, sont connus pour jouer un rôle majeur $[19,20]$. Le ciment est un matériau dont le pH de surface est fortement basique ( $\mathrm{pH} 12-13)$, ceci peut donc représenter un obstacle au développement des biofilms pour une grande majorité des microorganismes. Ainsi, aucune croissance bactérienne n'a été observée en bouillon
TSB ajusté à des pH supérieurs à 8, pour les trois souches sélectionnées cultivées en bouillon nutritif (Fig. 3).

Parallèlement, il a été constaté qu'une heure d'immersion des pastilles de ciment dans le milieu TSB entraînait une baisse significative $\mathrm{du} \mathrm{pH}$ mesuré à la surface du matériau (de $\mathrm{pH} 12$ à $\mathrm{pH}$ 9). Ce phénomène, probablement dû à l'effet tampon du bouillon, pourrait expliquer que la formation des biofilms soit possible sur les surfaces cimentaires comme cela a été observé pour chacune des trois souches de l'étude (Fig. 4). De plus, ce sont principalement les cellules en contact avec la surface cimentaire qui sont impactées par le $\mathrm{pH}$ basique du support, ne représentant qu'une faible proportion du biofilms total, la majorité étant en contact avec le milieu de culture dont le $\mathrm{pH}$ est de 7,6 .

\subsubsection{Vers une nouvelle méthode de quantification des biofilms « cimentaires »}

Plusieurs critères peuvent être étudiées afin de caractériser la morphologie d'un biofilm (profil de recouvrement des bactéries, biovolume, hauteur moyenne des micro-colonies, rugosité...) [17]. Cependant, la quantification d'un biofilm consiste habituellement à mesurer son biovolume et son recouvrement de surface en plusieurs zones puis d'en réaliser la moyenne. Les biovolumes moyens ainsi calculés pour les souches Pf SBW25 ViscA, Ec K12 et Bt 407, respectivement de $6,06 \times 10^{7}$, et $6,96 \times 10^{7}, 5,15 \times 10^{7} \mu \mathrm{m}^{3}$, apparaissent très proches, nous avons également observé une mortalité modérée, malgré les $72 \mathrm{~h}$ de croissance, de l'ordre de $7 \times 10^{5}, 4,0 \times 10^{5}$ et $6,3 \times 10^{5}$. Cependant comme l'illustre la Figure 4, la répartition des biofilms à la surface des pastilles de ciment n'est pas homogène, notamment pour les souches $E c$ K12 et Bt 407. Le simple calcul du biovolume moyen n'a pas permis de mettre en évidence cette hétérogénéité du biofilm. Afin de mieux la prendre en compte, nous proposons dans cette étude une méthode novatrice : la méthode SMART (Système de Mesure Appliqué au Recouvrement des biofilms cimenTaires) dont le principe consiste à (i) mesurer le pourcentage de recouvrement $(R \%)$ du biofilm pour 25 positions prédéterminées et régulièrement réparties (Fig. 1), (ii) affecter chaque valeur de $R \%$ obtenue à sa classe de recouvrement correspondante $(0-10 \%, 10-20 \%, \ldots, 90-100 \%)$, (iii) calculer et visualiser sous forme d'un profil (graphique), la représentativité de chaque classe (pourcentage d'image par classe/nombre total d'images) (Fig. 5). Par définition, un biofilm est dit homogène s'il est composé d'amas bactériens de tailles similaires (avec un fort ou un faible $R \%$ ) et répartis uniformément sur la surface du matériau. Les $R \%$ des 25 images obtenues se trouvent alors principalement repartis dans une classe et sont caractérisés par un pic majoritaire (Fig. 5A). Inversement, un biofilm hétérogène peut être défini comme étant composé d'amas bactériens de tailles variables et/ou non uniformément répartis sur la surface du matériau. Les $R \%$ des images obtenues sont alors répartis sur plusieurs classes, ce qui correspond à un profil composé de nombreux pics (Fig. 5B). 

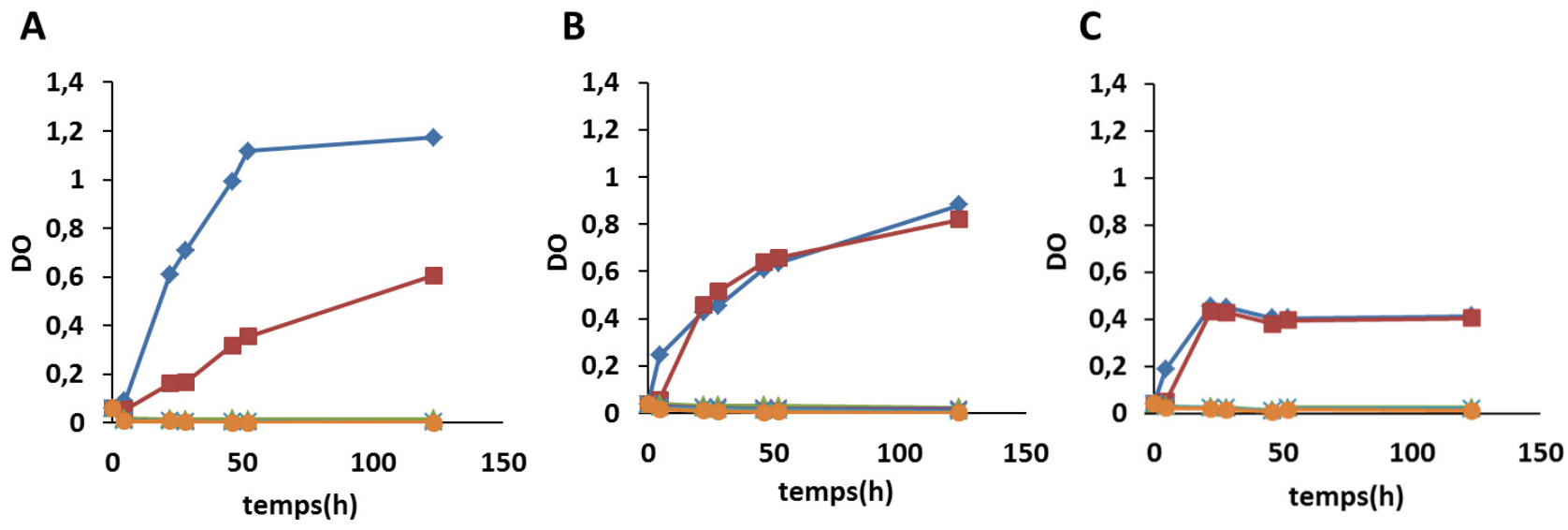

$\multimap-\mathrm{pH} 7 \multimap-\mathrm{pH} 8 \multimap \mathrm{pH} 9 \multimap \mathrm{pH} 10 \div \mathrm{pH} 11-\mathrm{pH} 12$

Fig. 3. Effet du pH sur la croissance bactérienne.

Effet de l'augmentation du pH sur la croissance bactérienne des souches (A) Pf SBW25 ViscA, (B) Ec K12 et (C) Bt 407 . La croissance bactérienne est réalisée à $25^{\circ} \mathrm{C}$, dans du bouillon TSB tamponné à differents $\mathrm{pH}$.

Fig. 3. Effect of $p H$ on bacterial growth.

Effect of increasing pH on bacterial growth of the strains (A) Pf SBW25 ViscA, (B) Ec K12 and (C) Bt 40\%. Bacterial growth was realized in $T S B$ broth adjusted to relevant $\mathrm{PH}$, at $25^{\circ} \mathrm{C}$.
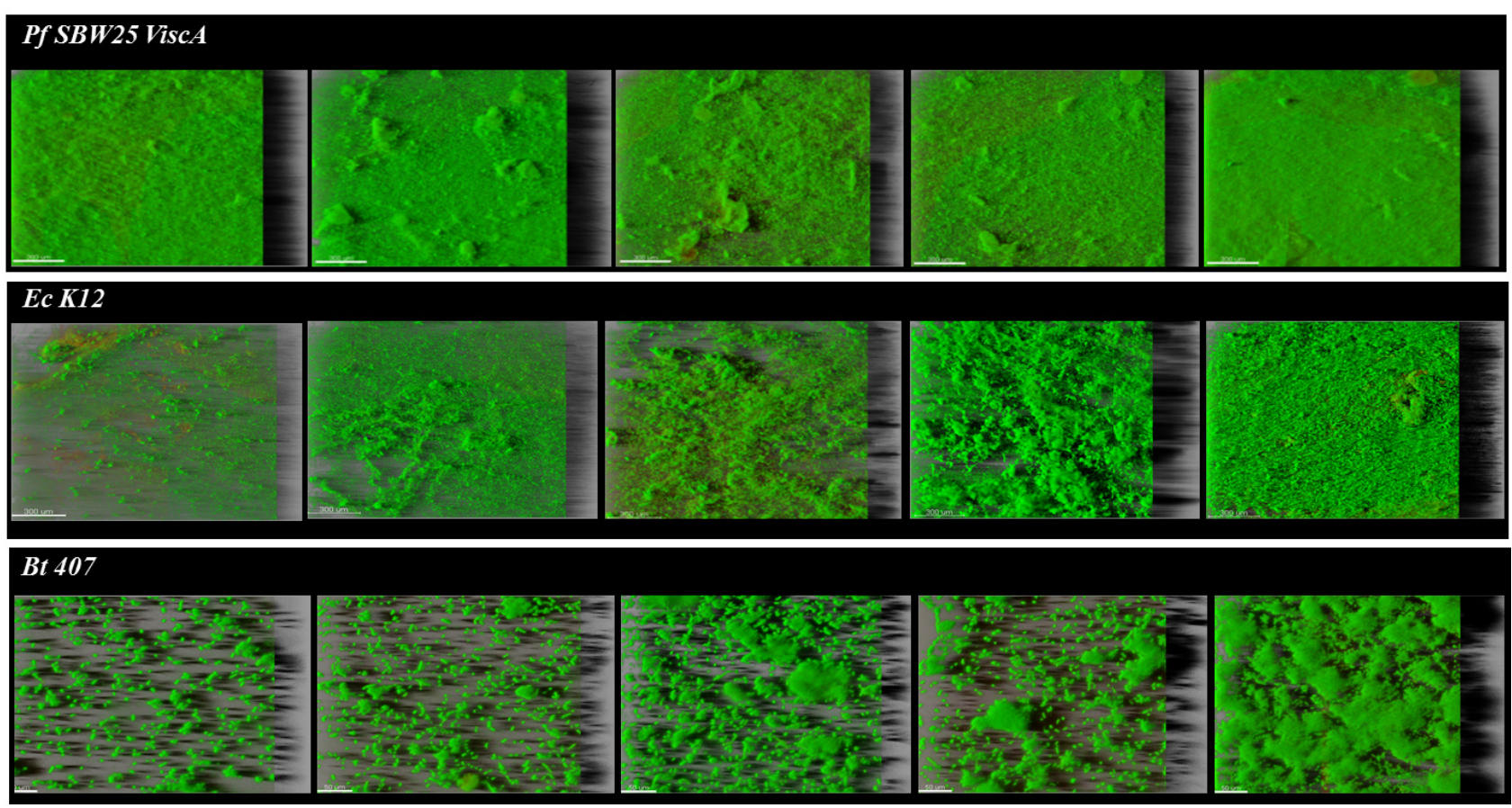

Fig. 4. Formation de biofilms bactériens sur des surfaces en ciment.

Visualisation de l'hétérogénéité des biofilms pour les souches Pf SBW25 ViscA, Ec K12 et Bt 407 par MCBL. Les biofilms ont été formés sur des pastilles de ciment, sur lesquelles cinq zones représentatives de chaque échantillon ont été sélectionnées et analysées par le logiciel IMARIS. La barre d'échelle correspond à $50 \mu \mathrm{m}$.

Fig. 4. Formation of bacterial biofilm on concrete surfaces.

Visualization of biofilms heterogeneity of Pf SBW25 ViscA, Ec K12 et Bt 407 by CLSM. Biofilms were formed on concrete and five representative areas were selected and analyzed by IMARIS software to each tree strains. Scale bar correspond to $50 \mu m$. 


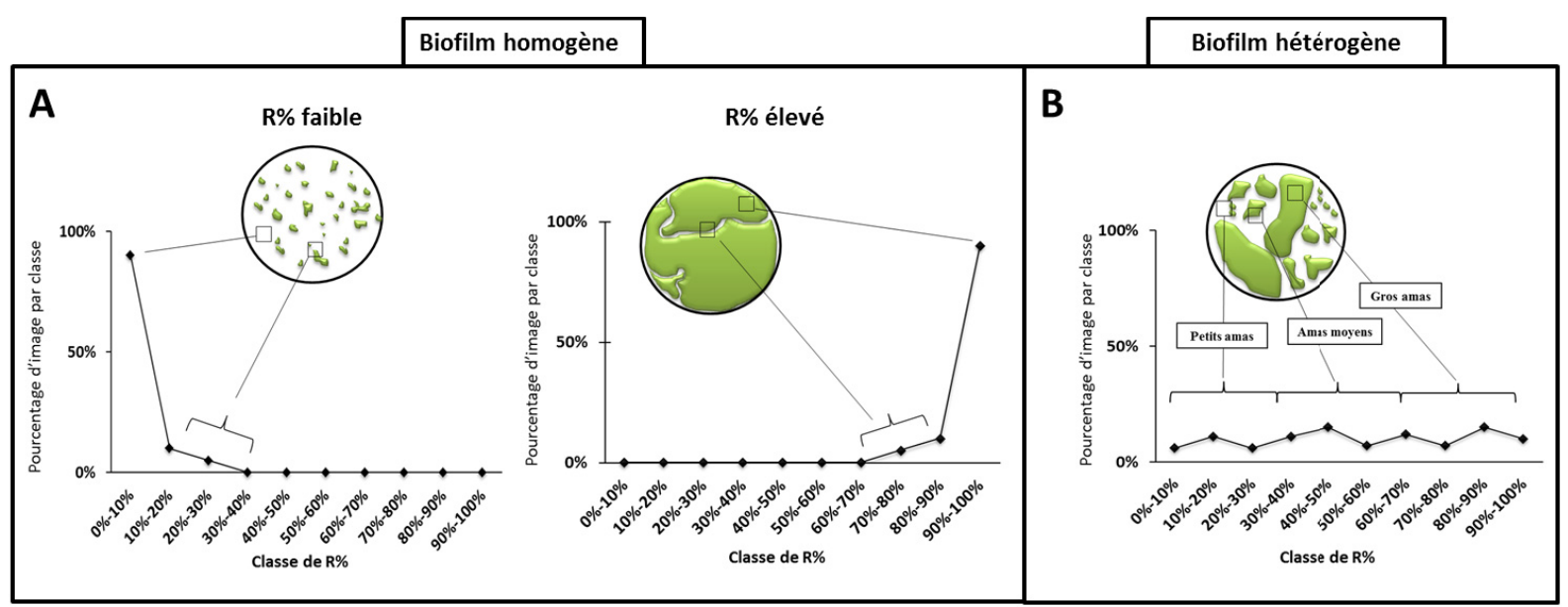

Fig. 5. Principe de représentation graphique de la méthode SMART.

Représentation schématique des biofilms homogènes $(\mathrm{A})$ et des biofilm hétérogènes $(\mathrm{B})$. Après analyse du pourcentage de recouvrement de surface $(R \%)$ des 25 champs d'observation sectionnés, le pourcentage d'images par classe est déduit (Nombre d'images par classe/nombre total d'images). Le profil du biofilm est obtenu sous la forme d'un graphique représentant le pourcentage d'images correspondant à chaque classe de $R \%$.

Fig. 5. Principle of graphical representation of the SMART method.

Schematic representation of two homogeneous biofilms (A) and one heterogeneous biofilm (B). After the analysis of the percentage surface covering (R\%) of the 25 observation areas selected, the percentage of images by class is deduced (Number of images per class/total image number). The graph represents the biofilm profile corresponding to the percentage of images in function of the $R \%$ class.

La méthode SMART détermine ainsi efficacement l'hétérogénéité des biofilms mais ne permet pas de quantifier leurs biovolumes (Bv). Afin de compléter la méthode et de quantifier l'hétérogénéité du biofilm, un stack a donc été réalisé sur cinq zones d'observation, chacune appartenant à l'une des 5 classes majeures de recouvrement. Les biovolumes calculés à partir de ces stacks sont alors pondérés par la représentativité de chaque classe $(\%$ classe $x$ ) et leur somme constitue le biovolume pondéré moyen du biofilm $(\mathrm{BPM})(\mathrm{BPM}(\mathrm{Bv} 1 \times \%$ classe 1$)+$ $($ Bv2 $\times \%$ classe 2$)+(\operatorname{Bv} 3 \times \%$ classe 3$)+(\operatorname{Bv} 4 \times \%$ classe 4$)+(\operatorname{Bv} 5 \times \%$ classe 5$))$.

Les profils ainsi obtenus pour les biofilms des trois souches étudiées, ont révèlé des caractéristiques d'une part très différentes en fonction des souches et d'autre part reproductibles pour une même souche (écarts type $<0,07$ ) (Fig. 6). Ainsi les biofilms formés par Pf SBW25 ViscA sont homogènes comme l'atteste la présence d'un seul pic pour les classes de recouvrement les plus élevées (Fig. 6A). Ce profil caractérise donc la forte contamination de la surface par le biofilm $(R \%>70 \%$ pour $90 \%$ du biofilm). Les biofilms d'Ec K12 sont caractérisés par une proportion élevée de zones fortement recouvertes ( $R \%>70 \%$ pour $70 \%$ du biofilm) cependant la présence d'un pic étalé dans les $R \%$ élevé démontre l'existence d'amas de tailles variables dénotant une hétérogénéité plus importante des biofilms d'Ec K12 (Fig. 6B). Une hétérogénéité encore plus marquée a pu être mise en évidence pour les biofilms de Bt 407 (Fig. 6C), comme le révèlent les valeurs de $R \%$ similaires obtenues quelle que soit la classe considérée $(R \%>50 \%$ pour $79 \%$ du biofilm) à l'exception des classes les plus extrêmes
( $R \%>90 \%$ pour $4 \%$ du biofilm). La technique SMART proposée, grâce à l'établissement de profils spécifiques et représentatifs des biofilms, permet donc de mettre en évidence des différences structurales majeures de ceux-ci pour les trois souches considérées.

Si l'on compare chacune des valeurs de BPM obtenues aux valeurs de biovolumes moyens des trois souches (Fig. 7), on note une meilleure adéquation des valeurs de $\mathrm{BPM}$ avec les images des biofilms obtenues en microscopie confocale (Fig. 4). En d'autres termes, la pondération des valeurs de biovolumes par la représentativité de chaque classe de $R \%$ permet une estimation plus réaliste de la biomasse des biofilms étudiés.

L'ensemble des résultats montre que le profil des biofilms obtenus par la méthode SMART ainsi qu'une estimation réaliste de la biomasse hétérogène (biovolume pondéré) sont nécessaires pour comparer et caractériser plus justement les biofilms. De plus, le choix d'autres variables de quantifications des biofilms telle que la porosité auxquelles serait appliquée la méthode SMART devrait permettre d'affiner la caractérisation des biofilms.

\section{Conclusion}

L'étude, à l'échelle microscopique, des biofilms implantés sur des surfaces hétérogènes comme les matériaux cimentaires, s'avère relativement délicate du fait des contraintes de tailles et de rugosité de ces matériaux. Les méthodes de quantification classique de biofilms sur des surfaces inertes reposent le plus souvent sur le calcul du biovolume moyen et de ce fait ne prennent pas 

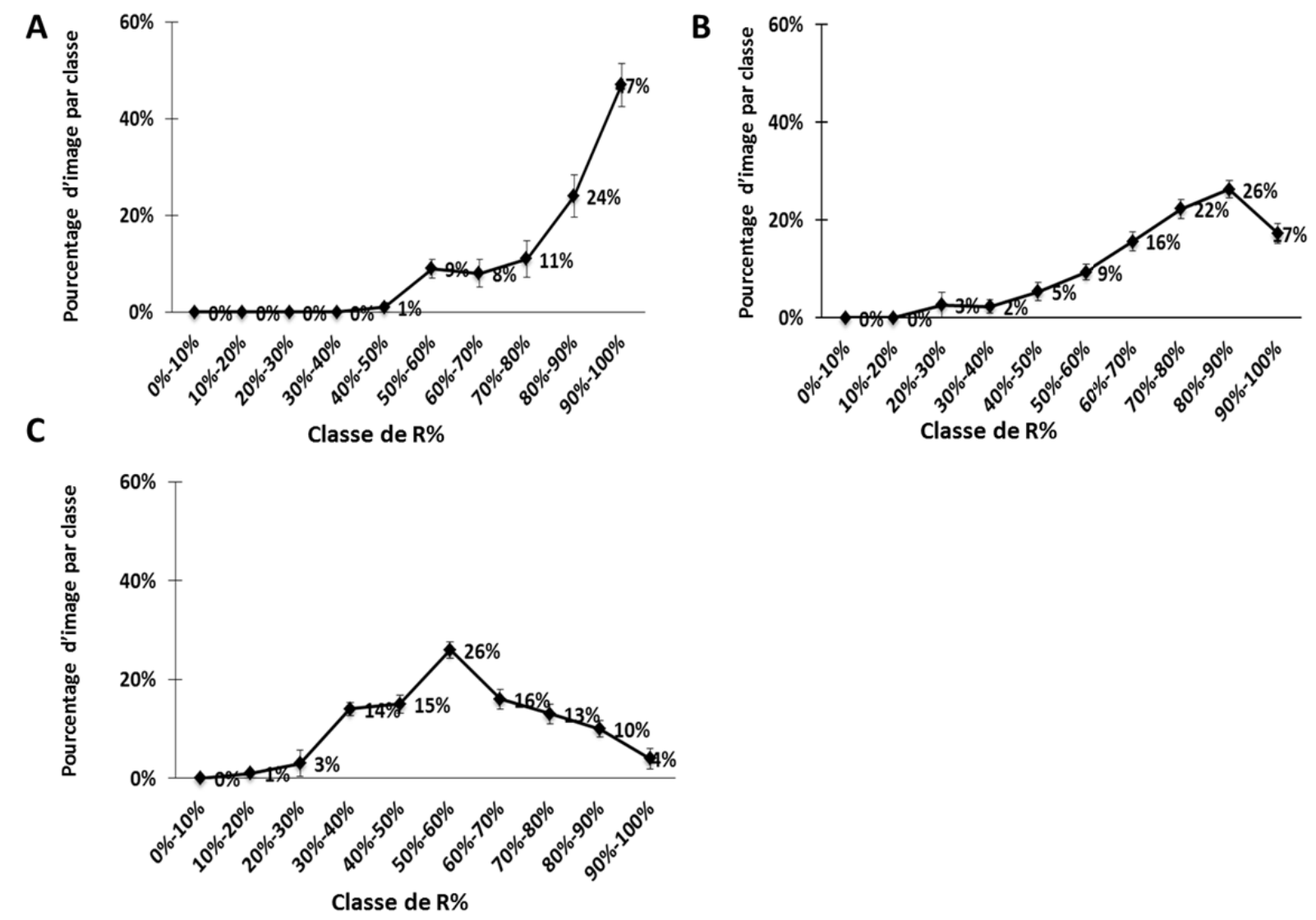

Fig. 6. Profil d'hétérogénéité des biofilms bactériens à la surface des pastilles de ciments.

Représentation graphique des profils d'hétérogénéité obtenus par la méthode SMART pour les biofilms bactériens de (A) Pseudomonas fluorescens SBW25 ViscA (B) Escherichia.coli K12 et (C) Bacillus thuringiensis 407 Le pourcentage d'images par classe représente le nombre d'images par classe par rapport au nombre total d'images.

Fig. 6. Profile of heterogeneity of bacterial biofilms on concrete surfaces.

Graphical representation of the bacterial biofilms heterogeneities profiles of (A) Pseudomonas fluorescens SBW25 VISCA (B) Escherichia.coli K12 and $(C)$ Bacillus thuringiensis 40\%, according to SMART method. The percentage of image per class represents the number of images per class in function of the total number of images.

en compte l'hétérogénéité de la structure de ces biofilms. Cette étude portant sur la formation de biofilms à la surface de matériaux cimentaires a en effet révélé une forte hétérogénéité de recouvrement difficilement quantifiable par la méthode classique. Pour y remédier, la méthode SMART mise au point a permis de représenter de façon réaliste et répétable l'hétérogénéité des biofilms obtenus avec les souches Pseudomonas fluorescens SBW25 ViscA, Escherichia. coli K12 et Bacillus thuringiensis 407 grâce à l'établissement d'un profil basé sur la classe de pourcentage de recouvrement de la surface cimentaire. Le calcul du «biovolume pondéré moyen » basé sur les classes majeures mises en évidence par ce profil, a permis d'estimer précisément la biomasse présente à la surface du matériau complétant ainsi l'analyse structurale. Les profils obtenus par la méthode SMART sont spécifiques des souches bactériennes étudiées.

La méthode SMART est spécifiquement adaptée aux cas de biofilms présentant une certaine hétérogénéité dans leur structure et/ou dans l'occupation des surfaces où ils se développent. Les matériaux cimentaires de par leur composition et leur forte hétérogénéité en sont un parfait exemple mais cette méthode peut bien entendu être utilisée pour toute surface engendrant cette typologie de biofilms. Cette méthode discriminante constitue également un outil de choix pour étudier l'impact des conditions environnementales (température, le pH, le milieu de culture...) sur les caractéristiques tridimensionnelles des biofilms. La méthode SMART pourrait donc être utilisée pour caractériser plus finement des modifications de structure et d'hétérogénéité induites par les conditions environnementales des biofilms, notamment les modifications de surfaces colonisées afin d'évaluer la bioréceptivité de celles-ci.

Remerciements. Les auteurs remercient l'Agence Nationale de la Recherche (ANR) pour le soutien financier du projet SEPOLBE « Substances Extracellulaires Pour les BEtons » (ANR-12-CDII-0004), les pôles de compétitivité Energivie, Hydreos et Advancity pour leur parrainage, ainsi que la plateforme MIMA2 pour les analyses microscopiques réalisées. 


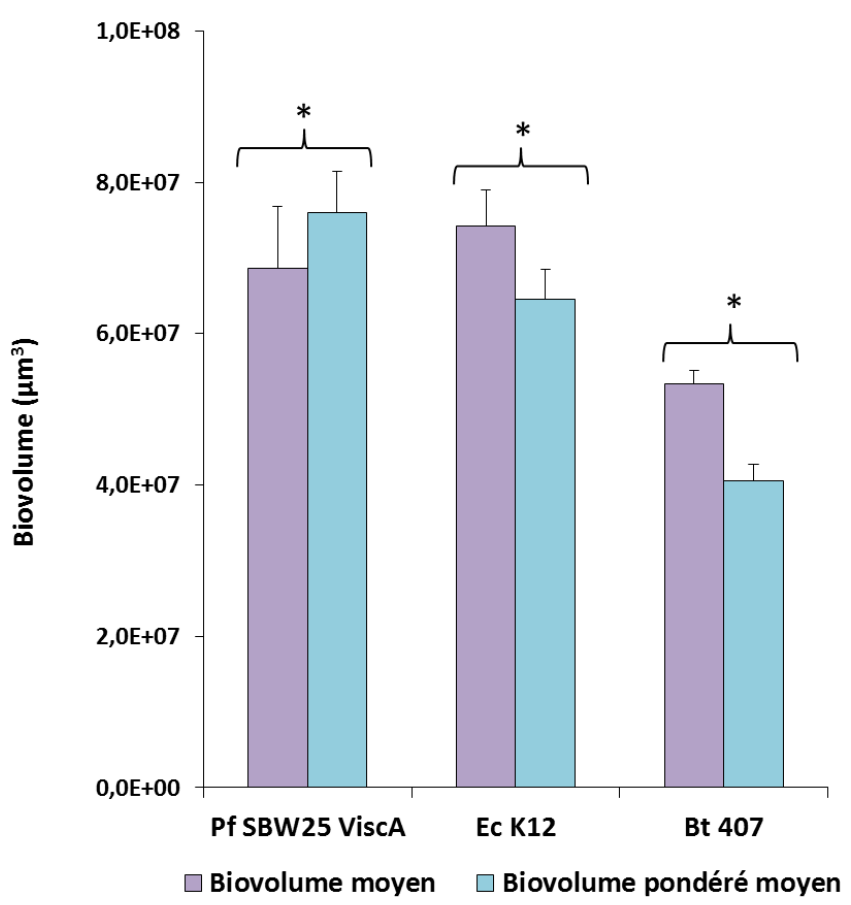

Fig. 7. Comparaison des biovolumes moyen et des biovolumes pondéré.

Comparaison de la méthode classique de quantification par calcul des biovolumes moyens avec la méthode des biovolumes pondérés moyens calculés par la méthode SMART. Le calcul des biovolumes par le logiciel PHLIP a été réalisé à partir de 5 séries d'images en Z (stack) représentatives des 5 classes majeures de $R \%$. Le biovolume moyen correspond à la moyenne des biovolumes obtenue pour les 5 stacks et le biovolume pondéré moyen est calculé en additionnant préalablement les biovolumes pondérés par la valeur de leur classe correspondante. ${ }^{*}{ }_{*}$ Significativité $=P<0,05$ (ANOVA).

Fig. 7. Comparison between mean biovolumes and weighted biovolumes.

Comparing the classical method of quantization by means biovolumes with the weighted biovolumes calculated by the SMART method. Biovolumes were calculating by Phlip software from five stack) representative of the five major classes of $R \%$. The mean biovolume corresponds to the mean of biovolumes obtained with the 5 stacks and the weighted biovolume was calculated by adding the biovolumes weighted according to the images percentages of the corresponding class $R \%$. "*" Significance was defined as $P<0.05$ (ANOVA).

\section{Références}

[1] USEPA, (United States Environmental Protection Agency) State of Technology: Review Report on Rehabilitation of Wastewater Collection and Water Distribution Systems. EPA/600/R-09/048. Cincinnati, $\mathrm{OH}$ : Office of Research and Development, 2009

[2] B. Carpentier, D. Chassaing, Int. J. Food Microbiol. 97 (2004) 111-122
[3] G. Midelet, B. Carpentier, Appl. Environ. Microbiol. 68 (2002) 4015-4024

[4] G. Midelet, A. Kobilinsky, B. Carpentier, Appl. Environ. Microbiol. 72 (2006) 2313-2321

[5] A.L. Ling, C.E. Robertson, J.K. Harris, D.N. Frank, C.V. Kotter, M.J. Stevens, N.R. Pace, M.T. Hernandez, Environ. Sci. Technol. 48 (2014) 7357-7364

[6] V. Gomez-Alvarez, Methods Mol. Biol. 1147 (2014) 323340

[7] V. Gomez-Alvarez, R.P. Revetta, J.W. Santo Domingo, BMC Microbiol. 12 (2012) 122

[8] J.W. Santo Domingo, R.P. Revetta, B. Iker, V. GomezAlvarez, J. Garcia, J. Sullivan, J. Weast, Biofouling 27 (2011) 993-1001

[9] S. Wei, Z. Jiang, H. Liu, D. Zhou, M. Sanchez-Silva, Brazilian J. Microbiol. 44 (2013) 1001-1007 (publication of the Brazilian Society for Microbiology)

[10] A. Houry, M. Gohar, J. Deschamps, E. Tischenko, S. Aymerich, A. Gruss, R. Briandet, Proc. Natl. Acad. Sci. USA 109 (2012) 13088-13093

[11] A. Houry, M. Gohar, J. Deschamps, E. Tischenko, S. Aymerich, A. Gruss, R. Briandet, Proc. Natl. Acad. Sci. USA 109 (2012) 13088-13093

[12] A. Koza, P.D. Hallett, C.D. Moon, A.J. Spiers, Microbiology 155 (2009) 1397-1406

[13] P. Lacroix-Gueu, R. Briandet, S. Leveque-Fort, M.N. Bellon-Fontaine, M.P. Fontaine-Aupart, C. R. Biologies 328 (2005) 1065-1072

[14] J. Baron, J. Ollivier, A. Desdevises, M. Buil, C.H. Detriche, C. Vernet, R. Duval, A. Carles-Gibergues, M. Pigeon, M. Berube, H. Hornain, J. Estoup, A. Capmas, T. Dumas, J. Letourneux, La durabilité des bétons, Presses de l'école nationale des ponts et chaussées, 1992

[15] A. Bridier, F. Dubois-Brissonnet, A. Boubetra, V. Thomas, R. Briandet, J. Microbiol. Methods 82 (2010) 64-70

[16] C. Munzer, H. He, N. Serres, S. Roux, T. Meylheuc, A. Lecomte, F. Feugeas, Bioréceptivité des matériaux cimentaires - protocole de préparation et analyses des surfaces en fonction du temps, $2^{\text {èmes }}$ Rencontres de l'AUGC, Polytech, Orléans, 2014

[17] J.B. Xavier, D.C. White, J.S. Almeida, Water Sci. Technol. 47 (2003) 31-37

[18] A. Bridier, D. Le Coq, F. Dubois-Brissonnet, V. Thomas, S. Aymerich, R. Briandet, PloS One 6 (2011) e16177

[19] M. Kuehn, M. Hausner, H.J. Bungartz, M. Wagner, P.A. Wilderer, S. Wuertz, Appl. Environ. Microbiol. 64 (1998) 4115-4127

[20] C. Chagnot, A. Agus, S. Renier, F. Peyrin, R. Talon, T. Astruc, M. Desvaux, PloS One 8 (2013) e59386

[21] E. Giaouris, N. Chorianopoulos, G.J. Nychas, J. Food Protection 68 (2005) 2149-2154 